\title{
Elevated peripheral blood levels of CXCL10 are associated with the presence of diabetic polyneuropathy in subjects with type 2 diabetes
}

\section{Pilar Ascaso}

Endocrinology and Nutrition Service, Hospital Clínico Universitario de Valencia. Valencia. Spain

\section{Ana Palanca}

Endocrinology and Nutrition Service, Hospital Clínico Universitario de Valencia. Valencia. Spain. Institute of Health Research-INCLIVA, Valencia, Spain

\section{Sergio Martinez-Hervas ( $\nabla$ sergio.martinez@uv.es )}

Endocrinology / Nutrition Serv., Hospital Clínico Univ. de Valencia. Spain; Institute of Health Res.INCLIVA Valencia; Centro de Investigación Biomédica en Red/Diabetes y enfermedades metabólicas asociadas; Univ. of Valencia https://orcid.org/0000-0002-6775-2034

\section{María Jesús Sanz}

Institute of Health Research-INCLIVA, Valencia, Spain. Centro de Investigación Biomédica en Red Diabetes y enfermedades metabólicas asociadas (CIBERDEM). Barcelona, Spain. Department of Pharmacology, University of Valencia, Spain

\section{Juan F Ascaso}

Endocrinology and Nutrition Service, Hospital Clínico Universitario de Valencia. Valencia. Spain. Institute of Health Research-INCLIVA, Valencia, Spain. Centro de Investigación Biomédica en Red - Diabetes y enfermedades metabólicas asociadas (CIBERDEM). Ba

\section{Laura Piqueras}

Institute of Health Research-INCLIVA, Valencia, Spain. Centro de Investigación Biomédica en Red Diabetes y enfermedades metabólicas asociadas (CIBERDEM). Barcelona, Spain. Department of Pharmacology, University of Valencia, Spain

\section{Jose T Real}

Endocrinology and Nutrition Service, Hospital Clínico Universitario de Valencia. Valencia. Spain. Institute of Health Research-INCLIVA, Valencia, Spain. Centro de Investigación Biomédica en Red - Diabetes y enfermedades metabólicas asociadas (CIBERDEM). Ba

\section{Research}

Keywords: Diabetic peripheral neuropathy, type 2 diabetes, CXCL10, inflammation 
DOl: https://doi.org/10.21203/rs.3.rs-17164/v2

License: (c) (1) This work is licensed under a Creative Commons Attribution 4.0 International License. Read Full License 
The authors have withdrawn this preprint from Research Square 